Letter to Editor

iMedPub Journals

www.imedpub.com

DOI: $10.21767 / 2471-8173.100038$

\section{Peripheral Nerve Suicide Transport Generates a Reproducible SCI}

\section{Abstract}

Unlike many organs in the body, the brain and spinal cord are unable to promote regeneration. The brain has a limited capacity for adaptive plasticity, but lacks functional replacement of injured, diseased or damaged neural parenchyma. Novel approaches to treat CNS lesions are required, as current therapies are solely palliative and do not generate repair. Essentially all clinical breakthroughs come from pre-clinical research where the fundamental principal for scientific advancement is experimental reproducibility. We describe a simple and reproducible pre-clinical model of spinal cord injury that can provide a platform to develop strategies to promote neural repair and regeneration.

Keywords: Plasticity; CNS lesions; Spinal cord injury; Fickle-P; Suicide transport

Received: November 20, 2017; Accepted: December 10, 2017; Published: December 14, 2017

Current therapies are inadequate in current animal models of spinal cord injury (SCl) [1-7]. A common objective is to mimic clinical $\mathrm{SCl}$ by generating a chaotic wound through cord laminectomy followed by controlled impact [2]. Not only is the surgery slow and challenging, but variations can generate a spectrum of wounds, and small sample sizes fail the limits of the 'fickle- $P$ ' [5]. Not surprisingly, many results have proven irreproducible and fail to enter clinical trials [7]. In short, the impact trauma model is inadequate.

Spinal cord research could benefit from insights on experimental design gained through years of invertebrate genetics, where virtually all genes involved in mammalian neural development were first identified. The Drosophila embryo at mid-neurogenesis is $4 \mathrm{~mm}$, much less than the width of a mouse spinal cord. Thousands can be harvested daily and quickly genotyped using fluorescent markers, and unlike the mouse model dozens of nerve cords can be dissected in one surgery session. The historical success of Drosophila reflects a tool kit that includes short gestation time, large sample sizes and absolute reproducibility. Combining this with genetic analysis has allowed experimenters to focus on a single variable at a time. Each of these factors is missing in pre-clinical vertebrate $\mathrm{SCl}$ models. The theme common to all major advances in biological sciences, from bacteriophage and Drosophila genetics to molecular biology and genomics, is 'keep it simple'. This theme is missing in pre-clinical $\mathrm{SCl}$ models and that has hindered progress. The experimental approach of simplicity and reproducibility is desperately needed in $\mathrm{SCl}$ research $[8,9]$.

\section{Joanna E Pagano, Allison S Liang, Christopher A Chrzan and Randall D Mckinnon*}

\author{
Department of Surgery (Neurosurgery), \\ Rutgers-Robert Wood Johnson Medical \\ School, Piscataway, NJ, USA
}

\section{* Corresponding author: \\ Randall D. Mckinnon \\ झ mckinnon@rwjms.rutgers.edu}

Department of Surgery (Neurosurgery), Rutgers-Robert Wood Johnson Medical School, 675 Hoes Lane, R309, Piscataway, NJ 08854, USA.

Tel: (732) 235-4419

Citation: Pagano JE, Liang AS,

Chrzan CA, Mckinnon RD (2017) Peripheral Nerve Suicide Transport Generates a Reproducible SCI. Spine Res. Vol.3 No.3:18

Retrograde transport of a neurotoxin, a process termed "suicide transport" may present a more useful approach to generate a reproducible spinal cord injury [9]. We examined suicide transport to generate $\mathrm{SCl}$ in mice using the sciatic nerve (Sn) for toxin delivery, in order to target motor neurons. We focused on delivery of an inhibitor of phosphoinositide 3'-kinase (PI3'K), a key intracellular effector of cell survival. Wortmannin binds the catalytic domain of PI3'K, blocks PI3K activity at very low concentrations (IC50=5 $\mathrm{nM}$ ), and it has a very short half-life which we considered an advantage for focal targeted MN destruction [8].

We accessed the SN at mid-thigh and injected $100 \mathrm{ng}$ wortmannin; the surgery is quick, survival is $100 \%$ and $>80 \%$ of test animals developed an ipsilateral hind leg motor deficit while sham controls had no visible phenotype. We used a simple water exit test to assess motor function of control and injured animals. Wild type mice exited without hesitation $(2.1 \pm 0.8 \mathrm{sec}$, mean \pm S.D, 8 mice $n=71$ trials), while mice treated with wortmannin had a significant delay $(38.6 \pm 21,15$ mice $n=253$ trials, $p<0.001)$, a phenotype that persisted through 6 months. At 14 days post wortmannin, histology revealed a reduction of choline acetyltransferase positive motor neurons in the ipsilateral lumbar 
spinal cord, relative to the contralateral cord. We also detected an ipsilateral gliotic response including astrocyte activation and microglial recruitment. These findings indicate that Sn delivery of wortmannin induces a site specific CNS motor neuron injury, and also show that water avoidance was a consistent and reliable test for measuring the functional consequences.

The Sn has historically been used for neuropathic pain and peripheral nerve regeneration studies [6]. Sn injury models generate distinct phenotypes after nerve compression, severance and transection. In our model the sham surgery generated a mild phenotype, consistent with transient peripheral neuropathy including reversal of axonal transport [1].

Motor neurons in the spinal cord survive peripheral transection [10] due to tropic support from Schwann cells in the proximal nerve and from re-innervated muscle after chronic injury [4]. We did not observe motor deficits after delivery of a Wnt pathway inhibitor, demonstrating that loss of $\mathrm{MN}$ in our model

\section{References}

1 Bisby MA, Bulger VT (1977) Reversal of axonal transport at a nerve crush. J Neurochem 29: 313-320.

2 Cheriyan T, Ryan DJ, Weinreb JH, Cheriyan J, Paul JC, et al. (2014) Spinal cord injury models: A review. Spinal Cord 52: 588-595.

3 Dietz V, Schwab ME (2017) From the rodent spinal cord injury model to human application: Promises and challenges. J Neurotrauma 34: 1826-1830.

4 Gordon T, Tyreman N, Raji MA (2011) The basis for diminished functional recovery after delayed peripheral nerve repair. J Neurosci 31: 5325-5334.

5 Halsey LG, Curran-Everett D, Vowler SL, Drummond GB (2015) The fickle P-value generates irreproducible results. Nat Methods 12: 179-185.

6 Savastano LE, Laurito SR, Fitt MR, Rasmussen JA, Gonzalez Polo was wortmannin specific and likely represents suicide transport. We thus suggest that $\mathrm{Sn}$ suicide transport represents a valid experimental $\mathrm{SCl}$ model that is suitable for genetically tractable small mammals (rodents) and can be scaled for statistically significant sample sizes.

The reproducibility of the injury provides a strong platform on which strategies to promote neural repair and regeneration can be explored in a meaningful way. Ideally such studies would focus on individual and specific aspects of the traumatic $\mathrm{SCl}$ wound, such as immune suppression, cell replacement, neural plasticity and path-finding, and remyelination [3].

\section{Conflict of Interest}

The authors declare no conflict of interests.

\section{Acknowledgments}

Supported by a grant from the New Jersey Commissions on Spinal Cord Research (05-3047; 11-015).

V, et al. (2014) Sciatic nerve injury: a simple and subtle model for investigating many aspects of nervous system damage and recovery. J Neurosci Methods 227: 166-180.

7 Steward O, Popovich PG, Dietrich WD, Kleitman N (2012) Replication and reproducibility in spinal cord injury research. Exp Neurol 233: 597-605.

8 Walker EH, Pacold ME, Perisic O, Stephens L, Hawkins PT, et al. (2000) Structural determinants of phosphoinositide 3-kinase inhibition by wortmannin, LY294002, quercetin, myricetin, and staurosporine. Mol Cell 6: 909-919.

9 Wiley RG, Blessing WW, Reis DJ (1982) Suicide transport: destruction of neurons by retrograde transport of ricin, abrin, and modeccin. Science 216: 889-890.

10 Xu QG, Forden J, Walsh SK, Gordon T, Midha R (2010) Motoneuron survival after chronic and sequential peripheral nerve injuries in the rat. J Neurosurg 112: 890-899. 